\title{
VALORES Y RACIONALIDAD EN LOS DIVERSOS TIPOS DE CULTURA
}

\author{
Javier San Martín \\ UNED - España \\ Centro de Filosofia da UL
}

Cuando uno recibe una invitación para un evento como este, siempre tiene la tentación de ofrecer algo ya pensado que no le va a costar mucho trabajo preparar, o aquello que a uno le gustaría poder ofrecer sobre el trabajo que está haciendo, o sobre lo que piensa hacer, o quiere ofrecer un adelanto programático de lo que está esperando un hueco en sus tareas para dedicarse a ello. En mi caso, ofrecí algo que me parecía que no me iba a dar trabajo porque en sustancia he escrito sobre ello, pero a la hora de llevarlo a cabo, he visto la enorme dificultad, que cuando lo escribí no percibí, porque operé un tanto ingenuamente, sin profundizar en los inmensos problemas que hay para abordar fenomenológicamente el tema. Ahora, ya con bastante historia detrás, me ha resultado más complicado y creo que no he sido capaz más que de ofrecer lo que podría llamar prolegómenos imprescindibles para el tema. La lectura constara de cuatro apartados. En el primero expondré lo que llamo el ethos filosófico. En el segundo, haré un relato necesario para enmarcar el tema y que lleva por título "La fenomenología de Husserl y la filosofia práctica". El tercero se centrará en el cambio en la fenomenología genética tomando en consideración la tendencia a la auto conservación. Y el cuarto tomará la auto identidad como punto de engarce de las regulaciones de la vida.

\section{El ethos filosófico}

Llegados a una altura de la vida, uno empieza a intentar ir encajando piezas de cara a una filosofía que pueda ser presentada de un modo más sistemático que puros intentos doxográficos. En mi caso todo viene regido por el 
ethos filosófico que en su momento descubrí en Husserl y que encaja perfectamente con el ideal de un joven de la generación del 68 , que ha pasado, además, por un seminario, del que le ha quedado, aparte de una vocación clara intelectual, un cierto afán de justicia que nos hace estar incómodos en un mundo radicalmente injusto. Ambas cosas se unen en el ethos filosófico de Husserl, que pude leer muy pronto, primero en el diario publicado por Walter Biemel; después en Erste Philosophie, tanto I como II, sobre todo en el artículo "Sobre una idea de cultura filosófica" (o europea), de 1922. Y ya más adelante, a partir de 1988, pero una vez centrado en una vocación filosófica decantada en un necesario diálogo entre la antropología y la fenomenología trascendental, en los textos que entonces pude leer, primero en Freiburg, ese curso, la Ética del 1920 y 1924, antes de que fuera publicada, y sobre todo la Einleitungvorlesung, del 1922-1923, con los "Londoner Vorträge", en los que Husserl especifica clara y literalmente el ethos filosófico.

Ahí adquirieron un importante papel los artículos para Kaizo, porque, en mi opinión, ellos, dentro de las humildes razones por las que Husserl los escribe, para paliar las estrecheces económicas por las que pasaba-resultado de las sanciones con que los ganadores de la guerra castigaron a Alemania, parecidas a las imposiciones a que ahora Alemania somete a los países europeos del sur y con tan funestos resultados presentes, sin que podamos prever un futuro cada vez más incierto- esos artículos, repito, tienen la doble ventaja, primero, que Husserl los escribe para un público no especialista en fenomenología, por tanto que está hablando a humanos que viven tal como vivimos en este mundo. Ahí diseña una antropología filosófica al margen de los problemas arquitectónicos que por entonces mismo estaba articulando al estudiar con precisión la relación del ser humano con el sujeto trascendental. $Y$ en ese texto, sobre todo en los artículos tercero y cuarto, expone el ideal humano, a cuyo servicio debe ponerse el ethos filosófico.

Porque la filosofia no es independiente, está para algo y por algo. Ese algo es la promoción de una humanidad auténtica. El ideal de humanidad auténtica es el que mueve la filosofía. En el cuarto artículo para Kaizo se sitúa ese ideal bajo algunas condiciones entre las cuales está la presencia de la filosofia para garantizar que el camino hacia la humanidad auténtica es seguro.

En estos años, 1920-1922, es cuando Husserl clarifica el ideal de la filosofia, y sobre todo la función que debe desempeñar en la vida humana. Y ya nunca dejará de pensar de ese modo. No sé si siempre pensó así, pero el ethos filosófico lo especifica por esos años. Por eso creo que hay que tomar los artículos para Kaizo como una especie de programa general en el que se especifica el modo como el ethos filosófico debe actuar. 


\section{Husserl y la razón práctica}

Mi ponencia, pues, se enmarca en el deseo de pensar la fenomenología desde el ethos filosófico en su función práctica. Y precisamente ahí es donde la relación de Husserl con la razón práctica resulta problemática, al menos si se toma la fenomenología desde su desarrollo histórico. Asumiría las conclusiones del denso trabajo de Agustín Serrano de Haro sobre la concepción husserliana de los actos básicos y los fundados (1995), que le llevarían a Husserl a proponer una primacía de la teoría sobre la práctica, porque ésta siempre estaría fundada sobre aquella. Pero el problema es que antes de la Guerra Husserl no parece tener conciencia explícita y profesional del problema inmenso que representaba la cultura europea y la profundidad de una crisis que solo la Gran Guerra reveló en toda su crudeza. En ese contexto se ve obligado o llevado a pensar en lo que la filosofía debe representar y la función que debe cumplir.

En mi lectura de Husserl, veo que la etapa de Gotinga está orientada a pensar la estructura de la fenomenología, y que la Gran Guerra le obliga a pensar en la función, ${ }^{1}$ para la cual tiene que profundizar en la historia de la filosofía porque no es viable pensar la función de la filosofía al margen del hecho cultural de su génesis. Por tanto, el pensamiento de la función, vinculado a la historia, es propio de la etapa de Friburgo.

Cuando hablamos de función, estamos hablando de lo que la filosofía pretende de cara al individuo y a la sociedad, por eso no están sobre la mesa cuestiones teóricas sino bien prácticas, porque es la sociedad y la vida humana en ella las que están en cuestión. La Guerra ha demostrado que los errores que a principios de siglo eran epistemológicos y a cuya solución se aprestaba la fenomenología, eran síntoma de una crisis mucho más profunda porque afectaban al ser humano entero. En La crisis de las ciencias europeas, al final de su vida, expondrá Husserl la frase que unifica la refutación del psicologismo con la crisis antropológica -una de cuyas manifestaciones más dramáticas en vida de Husserl fue la propia Guerra-, la frase de que si solo hay ciencias de hechos, no hay más que hombres de hechos.

En cierta medida, toda la filosofía práctica de Husserl estaría vinculada a este punto. Las tres citas que magníficamente jalonaron la intervención de Pedro Alves en Segovia en 2009 ( $c f$. Alves, 2011), las de Wundt y Kelsen

1 Sobre la estructura y función de la fenomenología, puede verse San Martín, "Estructura y función de la fenomenología de Husserl". Escritos de Filosofia, 21-22, Fenomenología - II, Academia Nacional de las Ciencias, Buenos Aires, pp. 47-66, Enero-Diciembre 1992. Ahora también como capítulo $\mathrm{V}$ del libro La fenomenología como estructura de una racionalidad fuerte. Madrid: UNED, 1994. 
directamente, la de Schmitt indirectamente, muestran que se pretende fundar todo, bien, en los hechos, como decía la frase de Wundt, bien en el hecho constituyente del legislador, que es fundador absoluto del derecho positivo, sin que quepa aducir más allá ningún criterio que legitime ese ato fuera del hecho de tener el poder de hacerlo. Pero incluso, la frase de Schmitt, de que los conceptos de orden jurídico no son sino los religiosos en otro formato: "La imagen metafísica que una determinada época se hace del mundo tiene la misma estructura que aquello que, sin más, la ilumina en cuanto forma de su organización política.", viene a decir que la razón práctica es un hecho religioso en el que no trasluce la autonomía de la libertad sino la irracionalidad de una decisión colectiva proveniente del pasado remoto, contra el que nada se podría hacer por ser las cosas como son. Así es como creo que se puede entender la frase que propone Schmitt: "Todos los conceptos centrales de la teoría moderna del Estado son conceptos teológicos secularizados" (2011: 13). Los hechos son lo que son.

Frente a ello, Husserl trata de reivindicar un ideal personal y social -ambos vinculados y garantizados por la filosofia, por una filosofía que se construye desde sus cimientos con rigor- como el momento definitorio más importante de Europa, y que esta ha formulado y debe reivindicar como su legado al mundo.

Pero en este contexto, la posibilidad de aplicar el rigor filosófico a la razón práctica es fundamental. O dicho de otro modo, la posibilidad de hacer una fenomenología rigurosa de la razón práctica es la base de toda la fenomenología. Y aquí es donde se oscurece el relato histórico sobre la fenomenología. Pues sin duda, por razones de un tipo u otro, esta filosofía pasó como una filosofía ajena a esa fenomenología de la razón práctica. Empecemos por decir que de las obras publicadas por Husserl la parte dedicada a la razón práctica estuvo casi ausente u oculta, primero en la intención misma de la fenomenología de una filosofia "científica", en la que esa palabra podía ser entendida desde lo "científico" de las otras ciencias, dando pie a la acusación a Husserl de un cientificismo que otros filósofos veían imposible e incluso indeseable. Segundo, en el concepto mismo del fenomenólogo como espectador desinteresado, por tanto, puro teórico que mira lo que ocurre sin importarle ni en una dirección ni en otra. Tercero, los análisis referidos a las estructuras relacionadas con la acción humana, por tanto, concernientes a la razón práctica, presentes en una cantidad considerable en las dos obras más importantes publicadas antes de la Guerra, las Investigaciones lógicas y las Ideas de 1913, empañaron o dificultaron la comprensión del lugar de la filosofía de cara a esa fenomenología de la razón práctica.

Más aún, desde ellos, resulta problemática la posibilidad misma de una fenomenología de la razón práctica. De hecho, hasta muy recientemente, 
los años 80 en adelante, la consideración de esa fenomenología de la razón práctica fue muy minoritaria. Así, mientras se veía que la ética era en Scheler su preocupación fundamental, para Husserl este papel lo tendría la teoría del conocimiento. Entre los éticos, Husserl no era ni siquiera mencionado. He comentado alguna vez (San Martín, 1992: 43; 2005: 17) que en la celebración del segundo centenario de la Crítica de la razón práctica se organizó en España un ciclo de conferencias sobre la incidencia de Kant en la posteridad. En él tuvieron su lugar comentarios sobre prácticamente todos los autores relevantes de la filosofía del siglo XX, todos menos Husserl.

Esta situación obviamente ha cambiado de modo radical a partir de los noventa, y en la actualidad resulta hasta difícil seguir la literatura al respecto. La publicación de los textos fundamentales de ética y otros ha sido decisiva. $Y$ justo en este momento podemos hacernos una idea de la importante evolución de Husserl en este terreno, en el que es fundamental distinguir la etapa de la fenomenología estática, que equivaldría grosso modo a la etapa de antes de la Guerra, es decir, las Investigaciones lógicas y las Ideas, y la etapa de la fenomenología genética, de después de la Guerra. ${ }^{2}$ Si para considerar el tema del idealismo fenomenológico la distinción de estas dos etapas es fundamental, en la consideración de la razón práctica aún lo es más. De todas maneras, hay que ver una considerable evolución de Husserl ya en la etapa de la fenomenología estática, ${ }^{3}$ desde las Investigaciones lógicas a las Ideas. Pero me parece que, sobre todo, son más importantes los cambios posteriores. El cambio de las Investigaciones lógicas a las Ideas consiste en que en la primera obra los actos relacionados con la práctica, la valicepción y los sentimientos que motivan la voluntad, son meras cualidades intencionales a las que no se podría preguntar por su razón porque esta se vehicula solo con la materia intencional que es teórica y no tiene relación con la práctica. ${ }^{4}$

En las Ideas el análisis es mucho más matizado, porque los atos intencionales valiceptivos son actos noéticos que tienen sus correlatos en el nóema, abriéndolos así a una fenomenología de la razón práctica. ${ }^{5}$ Como asume Mariana Chu en uno de sus brillantes artículos, "la cuestión se decide en las lecciones de ética de 1908/1909" (2012, nota 21). Lo cierto es que ya la Ética

2 Carlos Morujão me hizo saber que había un texto de esa época con notas claramente de análisis genético, pero en todo caso tomado el periodo en su conjunto creo que está dedicado de modo principal a la fenomenología estática.

${ }^{3}$ El artículo de Agustín Serrano de Haro tiene como objetivo mostrar la evolución de las Investigaciones lógicas a las Ideas.

4 Ver Agustín Serrano, ob. cit., p. 72 y nota 15.

${ }^{5}$ Aun con las dificultades que ve Agustín Serrano de Haro, para quien "el movimiento intencional del ánimo o de la voluntad ha de ser, pues, intrinsecamente feudatario" (ob. cit., p. 85), pero que le permiten una "interpretación alternativa" (p. 88). 
de 1914 opera con el objetivo de aplicar la refutación del psicologismo al campo de la axiología y la práctica. Pero de todos modos aún hay un problema proveniente de la fenomenología estática, pues pudiera ocurrir que esa fenomenología dificultara comprender en toda su profundidad el lugar de la práctica.

Me explico. En la fenomenología estática la fenomenología empieza por los actos teóricos, porque la propia filosofia es teórica. Agustín Serrano de Haro reconoce en el tratamiento de Husserl, incluso en las Ideas, el influjo de Brentano, para el que todo fenómeno psíquico o es una representación o se basa en representaciones. ${ }^{6}$ Este presupuesto brentaniano tiene un trasfondo ideológico importante, además de uno estático, porque toma al ser humano en un momento hecho de la vida, desde el que empezaría a describirla. Si tomamos un instante de la vida, tengo que empezar a decir que lo que tengo delante es un mundo a través de mis sentidos. La fenomenología empezaría de ese modo, avanzando en lo que iría llamando la estructura de la conciencia, $o$ de la vida, primero el conocimiento, luego la valoración y por fin la acción. Conocer, valorar/desear y hacer, son las tres grandes estructuras de la vida, que se corresponden con los tres vertientes de la razón, la teórica, la axiológica y la práctica.

El modelo es un humano maduro que se plantea un análisis de su vida, y empieza por la capacidad misma de conocer, el instrumento propio de la filosofía. Además, en el caso de Brentano, se trataba de un creyente enfrentado a las tendencias disolventes del positivismo. Husserl empieza en esa Escuela, y no disiente en cuestiones fundamentales.

Ahora bien, frente a Kant, en la fenomenología, y en la estela también de Brentano, entre la razón teórica y la razón práctica, se introduce la razón valorativa, porque no puede haber voluntad que no quiera algo, y ese algo tiene que ser estimado o valorado. ${ }^{7}$ El valor (o el ideal como valor deseado) está entre la teoría y la acción, entre el conocimiento y la voluntad. Y de ahí la relevancia de todo el campo de los sentimientos y emociones en la fenomenología que en Husserl adquiere máxima importancia ya en el segundo tomo de las Ideas, donde se describe todo el mundo de las personas en el que estas se

6 "Aquel primer supuesto [que la representación denota la categoria de las posiciones dóxicas] es el último rastro acrítico del principio brentaniano de fundamentación", ob. cit., p. 87.

7 Esta es la primera y dura crítica de Husserl a Kant en la Ética de 1920. Ver Husserl, Hua XXXVII, 214: "Denken wir es nun mit Kant auch aIs wollendes. Ich frage nun, ist es denkbar, dass ein Wollen frei sei von allem Werten, also allem FühIen? Ware ein solches Wollen nicht so widersinnig wie ein Ton ohne jede Tonintensitat oder eine Farbe ohne jede Ausbreitung oder eine Vorstellung ohne jedes Vorgestellte? A priori in unbedingter Wesensallgemeinheit muss also jedes Willenssubjekt ein wertendes, ein fühlendes Subjekt sein."Ver también San Martín, 1992, p. 71 y 2005, p. 27; M. Chu, 2012b, p. 244. 
mueven por motivación y que sería el mundo de los valores de todo tipo que determinan la voluntad. Así, Husserl, antes incluso de la Guerra del catorce, ya tiene muy claro que la crítica de la razón práctica kantiana está lejos de la realidad de las personas que se actúan motivadas en un mundo cultural. Por eso, es en este mundo cultural donde hay que centrar los estudios de la razón práctica, por más que la ética sea también una ética de principios, como el mismo Kant nos enseñó y Husserl se lo agradece y reconoce (Husserl, XxxVII, 199, 201, 232, 242; San Martín, 1992, 74).

Pero, desde las Ideas $I I$, el mundo de la cultura se convierte en el de las personas, porque en él desenvuelven éstas su vida. Esta vida está lejos de ser una vida que, según el modelo de la fenomenología estática, yo comienzo en un momento, en el momento en que la filosofia se decide a empezar. Precisamente, sobre todo después de la Guerra, Husserl desarrolla el complemento del método fenomenológico en la fenomenología genética que terminará dando un vuelco a los análisis anteriores, no porque los declare inexactos sino parciales.

\section{La vida humana como tendencia: fenomenología genética}

$\mathrm{Y}$ aquí me gustaría introducir un punto decisivo del artículo tercero de Kaizo, donde Husserl habla del punto de partida, que pone en la capacidad de autoconciencia y de inspeccionarse a sí mismo y en el autoconocimiento, autovaloración y autodeterminación (Hua XXVII, 23). Pero ese autoconocimiento, por tanto ejercicio de autoinspección, suponen la vida ya funcionando. Y si el filósofo debe partir de esa posibilidad indicada, es a partir del rasgo esencial de la vida de jugarse beständig in der Form des Strebens, de transcurrir "en la forma de la tendencia". Este me parece que es el punto clave del cambio de la fenomenología estática a la genética. En las lecciones de ética de 1920 y 1924 lo dice a la hora de resumir su ética al final: "Toda vida se ejecuta en sentido amplio en la tendencia y en ese sentido es práctica, se desarrolla en cuanto vida impulsiva (Triebleben), pasivamente, o activamente como propia vida de voluntad en atos del yo, mas en ello (wobei), las intencionalidades de valoración y de la esfera del conocimiento, por ambos lados y en una continua mezcla, son fundantes" (2004: 248).

En este contexto hay que tener en cuenta un principio metodológico procedente de la arquitectónica de la propia fenomenología: el humano es la auto objetivación del sujeto trascendental. Lo que diga de aquel con el correspondiente cambio, es decir, desobjetivándolo, me vale para este. ${ }^{8}$ Por tanto,

${ }^{8}$ Esta afirmación puede chocar, pero es un principio fundamental que se sigue del propio desarrollo de la fenomenología. Lo fundamental para la adscripción de una afirmación al humano y al sujeto trascendental es que provenga de la intuición, es decir, de la auto experiencia. 
aunque técnicamente en Kaizo se hable del Menschen, del ser humano, lo dicho vale para el sujeto trascendental. Por eso, es el sujeto trascendental el que vive en la forma de la tendencia. Nos valdría entonces el estudio de las tendencias hecho por Scheler y tan brillantemente resumido por Pilar Beites (2004: $173 \mathrm{ss}$.). Y es aquí donde hay que enmarcar toda la estructura de la vida, el conocimiento, la valoración y la acción. Todo son momentos de desarrollo de esa tendencia.

Por otro lado, no es de extrañar que en la fenomenología genética el problema de la animalidad tome toda su importancia porque nosotros estamos enraizados en la animalidad, y es nuestra animalidad la que nos da las formas originarias de las tendencias, los instintos que nos constituyen desde la base. En realidad, el conocimiento es una función subordinada a la vida en la forma de la tendencia a la auto conservación -y no repito a Ortega, sino a Husserl. $Y$ esta tendencia es anterior a cualquier desarrollo representativo. Desde ese momento hemos superado los estrechos marcos de la fenomenología estática porque es la preferencia la que dirige el conocimiento, aunque, cortando la vida en un instante determinado, nos pueda parecer que la preferencia viene después del conocimiento, dicho en términos más técnicos, que todo ato afectivo, toda percepción de un valor, o valicepcion, supone una materia representacional. Pero esta, en sus fundamentos, está subordinada a la tendencia, a la vitalidad, que es anterior al desarrollo de la vida.

Hay un texto del excurso de las lecciones de ética en que dice Husserl:

A saber, toda actividad supone una pasividad. Lo mismo que la actividad del conocimiento supone originariamente una pasividad del conocimiento, también una actividad valorativa supone una correspondiente pasividad. Esta es el sentimiento sensible que acompaña ya a la más primitiva donación y predonación del conocimiento, que colorea al más primitivo objeto de la percepción. Todo color sencillo, un tono, un olor, tienen, antes de toda actividad valorativa, un carácter afectivo fundado en algo que ya es pero nada afectivo. Si deconstruimos esa coloración afectiva, conseguimos objetividades del más profundo nivel. (xxxvII, 294)

Aún le parece este texto un poco confuso y añade un Beilage que va más allá:

Del mismo modo que el yo tiene un trasfondo de experiencia de lo ajeno al yo y una pasividad en la que tiene objetos de modo "inconscientes" que le afectan y que pueden convertirse en objetos del pensamiento y de la intuición, igualmente tiene el yo un trasfondo emotivo pasivo, a saber, los objetos afectan a la capacidad afectiva, en el trasfondo están coloraciones afectivas como una subconsciencia afectiva (Gefühlsunterbewusstsein), y estas afectan al yo a su modo. (xxxvil, 355).

El artículo mencionado de Pilar Fernández Beites es un intento de defender el alcance de la etapa estática husserliana, más allá de la etapa de antes 
de la Guerra. Lo bueno del texto es que toma nota del cambio que se da en Husserl al introducir los instintos, es decir, la vida animal, con lo que parecería romperse desde una perspectiva genética la tesis de la fenomenología estática. Toma en consideración el libro de Nam-in Lee sobre la vida instintiva en la que podría suponer la más clara superación de la tesis de la fenomenología estática porque el instinto proyecta tanto los campos de valoración como los cursos de acción, en definitiva, el sistema vital de preferencias, sobre los cuales se diseñan los cursos de conocimientos para concretar y ajustarse a la realidad. Pero no acepta la profesora madrileña las conclusiones del investigador coreano, por más basadas que estén en textos de Husserl, porque esas tendencias instintivas es cierto que, según ella, "tienen un papel decisivo en un plano genético, pues resultan fundamentales en los primerísimos momentos de la vida, mas este papel lo pierden posteriormente. $Y$, por tanto, en la vida adulta imperan las otras tendencias señaladas por Scheler, que, según vimos, ya sí se basan en actos objetivantes" (p. 186). Asume que la tesis de Nam-in Lee iría directamente contra esta propuesta y que hay textos de los manuscritos del último Husserl que darían la razón al intérprete coreano, porque los instintos serían "vivencias tendenciales, no basadas en actos objetivantes", que constituirían "la base de la vida psíquica y obligan a considerar falsa la tesis de la fenomenología estática que funda los actos no objetivantes en actos objetivantes" (ibid.). Después de rechazar por "inaceptable" la opinión husserliana de la "«sedimentación» de las experiencias de generaciones anteriores" (ibid.), por ser esta filogenética y esto no tener nada que ver con la experiencia individual, por ocurrir "por leyes físico-químicas" (ibid.), busca dar una solución asociacionista que parece desarmar totalmente nuestra animalidad afectiva, es decir, el mundo de la tendencia instintiva. No deja de ser ingeniosa la explicación que le lleva a pensar al lactante como un pequeño investigador a la búsqueda de "datos inmanentes" (p. 187) con los que construir "el objeto perceptivo" (p. 188), porque el "«impulso a percibir» es claramente «instinto»" de objetivación o de curiosidad. Parece olvidar que, en el niño lactante más bebé, estos movimientos están en función, ante todo, de la búsqueda de alimento, por tanto están movidos, ante todo, por la tendencia a alimentarse, antes que a constituir objetos teóricos.

Es imposible pensar la vida animal como constituida por movimientos arbitrarios sin sentido. Llama la atención que la canalización de esos movimientos arbitrarios hacia la constitución, por sintesis pasivas, de objetos tridimensionales sea capaz de eliminar las tendencias instintivas del hambre, la sed, la necesidad de cobijo en el regazo de otra persona y, por supuesto, más adelante ya, el despertar del sexo que nos lleva irremediablemente a fijarnos en las otras personas, generalmente del otro sexo, desde unas preferencias que incluso pueden venir modeladas antes de la educación. La vida animal ins- 
tintiva no desaparece nunca de nuestra vida, sólo que el desarrollo cultural en el que se inserta modela tanto las partes teóricas que darán contenido a esas tendencias instintivas primeras, como la identidad de los individuos que se convertirá ella misma en punto de preferencia para el resto de la vida. Porque la identidad del individuo, el saber quién soy, no es algo teórico, es el primer centro de afecto, porque cualquier puesta en cuestión de esa identidad nos conmueve y afecta.

\section{Auto identidad, cultura y regulaciones de la vida}

Lo que acabamos de decir nos sucede, primero, como animales. La primera alarma es el hambre y la sed, que han de ser vistos como afecciones en la identidad biológica, ambas son las primeras alarmas que motivan la búsqueda de alimento, que tiñen el mundo de un colorido específico. Más adelante, la identidad irá configurándose en las diversas fases en que se asume la identidad social, con sus problemas de ajuste con la identidad personal. Pero creo que la auto identidad es el punto del afecto básico que está detrás de todo el sistema de preferencias, pues nada nos afecta más que lo que pone en peligro la identidad. Por eso la tendencia básica que permanece a lo largo de la vida como la base que la articula es la tendencia a la auto conservación. La tendencia a la auto conservación, como el conato básico, es el modo como estamos en la vida, es el afecto básico que precede a todo conocimiento.

Más aún, lo que Husserl llama la actitud natural creo que debe ser interpretado como la seguridad básica con que nos sentimos en el mundo. No es una tesis teórica, un ato objetivante, o el ato de los atos objetivantes. Es el correlato de valor del mundo seguro, que se modula en lo que, con Antonio Zirión $(2009,141)$ llamaríamos "el colorido de la vida", según se nos vaya desarrollando esta de un modo u otro, en general o en cada momento o día.

Precisamente, este punto es el que yo considero decisivo en el Husserl genético, el paso de la animalidad instintual puramente animal a la vida humana asentada, sí, en una afectividad preteórica que da color a la vida, en esa especie de "subconsciencia afectiva" que subyace a toda otra actividad, y de la cual la llamada actitud natural es el primer asiento en el que nos movemos.

A partir de ahí, es la cultura la que va modulando los diversos niveles, pero ya en una urdimbre en la que se mezclan de modo inextricable afectos, valores, conocimientos y acciones, cada uno de los cuales motiva reacciones en los otros niveles, sin que, a una altura de la vida, podamos decir qué es antes y qué después, porque las acciones suscitan sentimientos que afectan al colorido de la vida, que a su vez desencadenará intereses que orientan el conocimiento, que, a su vez, puede descubrir valores, que suscitarán deseos, que a su vez pueden mover a la acción. 
Por tanto, tenemos una secuencia importante, tendencia a la auto conservación, como el punto de partida. Correlato de esta tendencia en la tesis ante todo afectiva de la seguridad del mundo, el mundo como nuestro hogar seguro. Desde esta base afectiva se dan las modulaciones de la auto identidad, en la cual ya la más amplia regulación, después o a la vez que la de los status, es la profesión.

El mundo seguro en el que vivimos no es un mundo natural, propio de las ciencias naturales, sino un mundo cultural, un mundo que de entrada está poblado de objetos culturales enmarcados en campos pragmáticos y que, por tanto, incorporan el sentido práctico de que sirven para una acción u otra, un mundo que es sedimentación, ante todo, de los sistemas de preferencias de nuestras generaciones anteriores.

Un punto más, ya para aterrizar, aunque más bien a modo de programa, en lo que anuncio en el título. Llama poderosamente la atención el hecho de que no nos hayamos esforzado en presentar de modo ordenado la totalidad del concepto de cultura en Husserl, cuando es posiblemente el filósofo que ofrece más desarrollos sobre este concepto, además, perfectamente integrado en su filosofía desde Ideas II. Es cierto que, antes de la publicación de Ideas II, sólo se podía tener un atisbo por el primer texto póstumo publicado, en este caso por Fink, lo que le da especial relieve, "Die Frage nach dem Ursprung der Geometrie als intentionalhistorisches Problem", o tal como lo conocemos, nosotros, "El origen de la geometría", publicado después como Beilage o Anexo III del tomo VI de Husserlaina, La crisis de las ciencias europeas. El texto póstumo salió ya en 1939. ${ }^{9}$ En él Husserl expone cuestiones fundamentales tanto para una filosofia de la cultura como para una filosofia de las ciencias humanas, si bien, en ausencia, en 1939, del texto de la tercera parte de La crisis, esos desarrollos resultaban un tanto enigmáticos y, en ausencia también de Ideas $I I$, dificiles de percibir en todo su alcance, pues en ese texto no está más que la definición formal de cultura (Hua VI, 379), y la indicación de dos de los tipos de cultura, la cultura ideal, explicada como opuesta a la cultura técnica. Aunque ya eso hubiera bastado para intentar una filosofía de la cultura desde la fenomenología, en esa publicación faltaba el tipo de cultura fundamental, que se nos ofrece precisamente en los artículos de Kaizo, que como se sabe no fueron conocidos hasta 1988, a saber, la cultura práctica, las regulaciones de la vida, que además son las que centran todo el interés práctico de la filosofia, de manera que, sin ese concepto de cultura, los otros dos, la cultura ideal y la cultura técnica, quedaban sin base.

${ }^{9}$ En la Revue Internationale de Philosophie, 2: 203-225. 
En efecto, las regulaciones de la vida constituyen el concepto fundamental en el que se centra y se debe centrar en la fenomenología, primero, el nivel emotivo y, segundo, el nivel práctico. Vamos a intentar ofrecer un desarrollo de estos dos puntos absolutamente nucleares de la fenomenología en su etapa genética, la desarrollada por Husserl, principalmente, en Friburgo. Tendría que responder a varias preguntas, todas sumamente interesantes en este camino, pero que, llegados a esta altura de mi exposición, no pueden sino anunciar un programa de investigación.

Primera, ¿en qué medida creo que las regulaciones de la vida centran el nivel fundamental emotivo de la vida? Segundo, ¿en qué medida en esa pregunta se centra el nivel práctico de la fenomenología? Respecto a la primera, está en relación con lo que hemos visto de la auto identidad. La auto identidad es el punto crucial de la vida, lo que hemos sido, somos y queremos ser es el núcleo que organiza nuestro mundo, y del que surgen todos los rayos de valoración. Precisamente, la actitud natural hemos dicho que hay que vincularla afectivamente a este ámbito de auto identidad. Auto identidad no es algo teórico, es auto afecto, y fuente de valoración. Aquello que hace peligrar la auto identidad será valorado negativamente. Y por suerte, no tenemos que esperar a objetivar el peligro, la vida animal tiene antenas de alerta para los peligros. En la vida social nos hemos tenido que entrenar, pero lo que siempre está abierto es la tendencia a la auto conservación de lo que somos y queremos ser. Esta tendencia es previa a cualquier objetivación, es el foco desde el que vemos el mundo y con el que vemos el mundo. Todo se integra a través de ese foco. La auto identidad tiene, naturalmente, varios niveles, el primero es el de la propia vida. Pero mi mundo vital no es un mundo de soledad, nunca estoy solo, desde niño he estado con otros, por eso mi auto identidad depende también de la vida de los otros, su sufrimiento o peligros son también míos. La capacidad de sintonizar con los otros es también una capacidad emotiva originaria, previa a cualquier objetivación. Hasta con los animales nos sintonizamos, lo mismo que ellos con nosotros. La expresión del rostro es antes que nada afectiva. Quiero decir que el mundo de los afectos constituye una capa originaria con la cual nos acercamos al mundo desde nuestra más temprana edad. Y ahí esta mi auto identidad en urdimbre con los otros, no con la auto identidad de los otros, a la que yo no tengo acceso, sino con los otros como personas que se expresan en su cuerpo. $Y$ aquí vienen las valoraciones positivas y negativas del entorno, con las que siempre percibiré el entorno. Siendo eso así, las regulaciones de la vida, las regulaciones de mi auto identidad, es decir, el conjunto de lo que soy y la previsión de antemano de lo que quiero ser, es la fuente fundamental para la valoración, primero, del mismo proyecto que quiero ser $\mathrm{y}$, segundo, de los medios que quiero emplear. 
$\mathrm{Y}$ aquí hay un punto importante que tener en cuenta. Lo que quiero ser queda mediado por lo que soy y por lo que puedo ser. Es decir, no todo lo que me gustaría ser puedo ser. La valoración, entonces, de lo que quiero ser está también en relación con lo que me gustaría ser pero no puedo ser. Según esa distancia, valoraré más o menos lo que quiero ser y las regulaciones de la vida.

En cuanto a la segunda. Hemos dicho que Husserl parte de la consideración de la vida como tendencia. La tendencia es a la auto conservación. Pero la previsión del futuro no es anárquica sino que se da en el marco de las diversas regulaciones previstas en la cultura por los estados y las profesiones. Lo que voy a hacer esta determinado por lo que soy y quiero ser. Soy lo que soy biológicamente y lo que la sociedad me dice que soy. Aquí se enraizan los status que se llaman en antropología cultural adscritos, y luego los adquiridos, que vienen determinados por lo que quiero ser, siempre de acuerdo a los moldes de identidades que están previstos en la sociedad y que abarcan fundamentalmente a los estados adscritos en la vida, por sexo o por "estado civil", y los adquiridos, fundamentalmente, en las profesiones. Son las profesiones las máximas regulaciones de la vida en las que envolvemos la auto identidad y por eso es una de las principales fuentes de valoración y acción de la vida.

Hasta aquí hemos dado sentido a la exigencia husserliana de que entre la voluntad y el conocimiento, entre el ámbito de la razón práctica y el de la razón teórica, está la vida con sus exigencias y la cultura que ha ido modelando las exigencias. Para terminar es necesario preguntarse en qué medida en esos ámbitos cabe hablar de razón, que es el objetivo al que debemos aspirar.

Y aquí debemos tomar en consideración lo que Husserl llama en Kaizo el verdadero "punto de partida". Pues si, para la definición o descripción de la vida, el hecho de la tendencia a la auto conservación es el punto de partida, para la filosofia, que es donde estamos, el punto de partida es la capacidad de auto inspección. Porque es en la auto inspección donde se ventila lo más importante de la vida humana, ya no en cuanto animal, sino en cuanto humana. Incluso no sería difícil reconducir los logros culturales, que redefinen la vida humana, a precisamente la capacidad de la auto inspección. Pero en la auto inspección hay que detectar dos planos, uno constituido por tres posibilidades, el autoconocimiento directo que llevamos con nosotros mismos procedente de la forma misma del tiempo; la valoración que llevamos con nosotros mismos, de nuestra autoestima; $y$, por fin, la autodeterminación porque continuamente estamos decidiendo qué hacer. Pero hay un segundo plano reflexivo en los tres niveles. Nos interesa sobre todo en el segundo, la autovaloración, que es la evaluación de nuestras acciones. Esta autoevaluación se hace en de acuerdo a dos posibilidades, en la primera nos referimos al proyecto de lo que queremos ser, en la segunda, al logro de eso que queremos ser. 
Una vez expuesto esto, no sería difícil aplicar los criterios de racionalidad a cada uno de los niveles. Razón es un título de legitimidad. Tener razón es estar legitimado. En el plano del conocimiento, que por lo general se da en el marco de la acción ordinaria en el mundo, es decir, en el desarrollo de los proyectos enmarcados en los campos pragmáticos, y en el saber enciclopédico que la sociedad va acumulando, la razón está en la legitimidad, es decir, en la donación originaria de aquello a que aplicamos el título de racional. No hay ninguna dificultad de comprender esta racionalidad. En el plano de los valores, la mayoría de estos se da en el marco del ajuste en los campos pragmáticos, la legitimidad de un medio ajustado al fin perseguido. Por tanto, en la cultura ideal y en la técnica no hay dificultad en la exposición de los títulos de legitimidad. El problema de la razón radica en el ámbito de la cultura práctica, que es el repositorio de las regulaciones de la vida que asumimos para ser lo que queremos ser y que quedan sometidas a la auto inspección.

Aquí la legitimidad está, primero, en el proyecto mismo que tenemos. Pero para evaluar el proyecto no tenemos más remedio que introducir algún criterio que permita concluir que una profesión no nos puede defraudar o decepcionar. Si su logro nos decepciona, es que no tenía plena legitimidad, o no cumplía todos los requisitos para esa plenitud, y es en este contexto en el que Husserl establece la comparación de las regulaciones de la vida, la comparación de las profesiones, al preguntarse bajo qué condiciones son legítimas, es decir, no decepcionantes, aunque solo sea como posibilidad. Y este es justo el lugar de inserción de la ética, como el modelo de ser humano que mide todo el resto de profesiones, todas las cuales pueden decepcionarme, en la medida en que no se midan por el proyecto ético. $Y$ en el proyecto ético los otros se hacen presentes desde la seguridad de que mi bien está mediado por el suyo, pues partiendo de que el proyecto de vida está orientado hacia el cumplimiento de una vida plena, no hay posibilidad de ese cumplimiento en un mundo en el que los otros son desgraciados.

En la ética de Husserl los humanos llevamos con nosotros, además del proyecto profesional, que es nuestra profesión, otra profesión, que es la de ser buena persona, el proyecto ético. Este es el que mide todos los demás, tanto desde una perspectiva axiológica como desde una perspectiva práctica. La legitimidad o racionalidad en la cultura, en Husserl, depende, por tanto, de la racionalidad del proyecto ético. 


\section{Referencias}

Alves, Pedro M. (2011). "Razón práctica. Reflexiones husserlianas". Investigaciones Fenomenológicas, vol. monográfico 3: Fenomenología y politica (2011), pp. 11-33.

BIEMEL, Walter (1956). "Edmund Husserls persönliche Aufzeichnungen", editado por W. Biemel, en Philosophy and phenomenological Research, XVI (1956): 293-302.

CHU, Mariana (2012a). "La objetividad de los valores en Husserl y Scheler. Una disputa fenomenológica". Séptimas Jornadas Peruanas de Fenomenología y Hermenéutica, sin paginación y OPO IV Segovia (en prensa).

Chu, Mariana (2012b). “¿Afectan las objeciones de Scheler a Kant también a la ética de Husserl?". Acta fenomenológica latinoamericana. Volumen IV (Actas del $V$ Coloquio Latinoamericano de Fenomenología). Lima: Círculo Latinoamericano de Fenomenología / Pontificia Universidad Católica del Perú, pp. 241-255.

Fernández Bertes, Pilar (2004). "Cosas, valores y tendencias. Husserl frente a Scheler", en Escritos de Filosofia, 44 (2004): 163-190.

HusserL, E. (1989). Aufsätze und Vorträge (1922-1937). Husserliana XXVII, T. Nennon y H. R. Sepp (Hrsg.). Dordrecht: Kluwer Academic Publishers.

HusserL, E. (1989). "Fünf Aufsätze über Erneuerung", Aufsätze und Vorträge (19221937). Husserliana XXVII, T. Nennon y H. R. Sepp (Hrsg.). Dordrecht: Kluwer Academic Publishers, pp. 3-122. Hay traducción al español, Renovación del hombre y de la cultura. Cinco ensayos. Introducción de Guillermo Hoyos Vásquez. Traducción de Agustín Serrano de Haro. Editorial Anthropos y UAM, Iztapalapa, México, 2002.

HusSERL, E. (2004). Einleitung in die Ethik. Vorlesungen Sommersemester 1920/1924, Husserliana XXXVII, edición de Henning Peucker. Dordrecht: Kluwer Academic Publishers, 2004.

SAN MARTín, Javier (1992). "Ética, antropología y filosofía de la historia. Las Lecciones de Husserl de Introducción a la ética del Semestre de Verano de 1920". Isegoría 15 (1992): $43-77$ (también, cap. VIII de La fenomenología como teoría de una racionalidad fuerte, UNED, Madrid, 1994.

SAN MARTín, Javier (2005). "El legado de Kant". In: Ana M. Andaluz Romanillos (Ed.), Kant. Razón y experiencia. Salamanca: Publicaciones de la Universidad Pontificia de Salamanca, pp. 15-29.

Serrano de Haro, Agustín (1995). “Actos básicos y actos fundados. exposición crítica de los primeros análisis husserlianos". Anuario Filosófico, 1995 (28): 61-89.

ZRrón, A. "El resplandor de la afectividad". Acta fenomenológica latinoamericana. Volumen III (Actas del IV Coloquio Latinoamericano de Fenomenología). Lima: Círculo Latinoamericano de Fenomenología / Pontificia Universidad Católica del Perú; Morelia (México), Universidad Michoacana de San Nicolás de Hidalgo, pp. 139-153 


\begin{abstract}
The author deals with philosophical ethos in its practical function. After the First World War Husserl not only considers the structure but also the function of phenomenology. A meditation on this function, with regard to the history of philosophy, is his primal task in the Freiburg years. Thus the possibility of developing a rigorous phenomenology of practical reason becomes the basis of phenomenology. The importance of Husserl's characterization of life in terms of striving is stressed as a key motive in the turn from static to genetic phenomenology. It is argued that instinctive life is inserted in a cultural development that shapes a theoretical side providing content to our primal tendencies as well as the identity of individuals. A decisive point in genetic phenomenology is the passage from instinctive animality to human life. This development rests on a pretheoretic affectivity that colors life as a sort of subconsciousness underlying all our activities. It is the originary stratum through which we approach the world. The article goes on to high-light the importance of Husserl's concept of culture and specifically of his practical concept of culture which is made up of life-regulations and without which an ideal and a technical culture are devoid of a ground. Finally, the author considers life-regulations as a key problem to be dealt with by phenomenology first in the emotional and then in the practical level.
\end{abstract}

